\title{
Tight-binding investigation of the metallic proximity effect of semiconductor-metal double-wall carbon nanotubes
}

\author{
Jie $\mathrm{Lu}^{1}$ and Shidong Wang ${ }^{2}$ \\ ${ }^{1}$ Physics Department, The Hong Kong University of Science and Technology, Clear Water Bay, Hong Kong SAR, China \\ ${ }^{2}$ Theoretische Physik, Universität Regensburg, 93040 Regensburg, Germany
}

(Received 19 April 2007; published 20 December 2007)

\begin{abstract}
Based on a well-known tight-binding Hamiltonian formulation of carbon nanotubes, the local density of states of semiconductor-metal double-wall carbon nanotubes (DWCNTs) is evaluated numerically. The results verify that the semiconductor shells in contact with the nearby metallic shells become metallic, a direct evidence of a metallic proximity effect. This proximity effect can be used to explain the anomalous field effect characteristics observed in the field effect transistors made of the semiconductor-metal-type DWCNTs.
\end{abstract}

DOI: 10.1103/PhysRevB.76.233103

PACS number(s): 73.63.Fg, 73.20.At

Quantum wires have attracted much attention in recent years for their novel properties ${ }^{1-6}$ and for their great relevance to the nanotechnologies. In particular, carbon nanotubes (CNTs) are among the mostly studied systems because of their fantastic physical properties ${ }^{7-16}$ and potential applications in devices ${ }^{17}$ and wiring ${ }^{18}$ in nano- and/or moleculeelectronics. A CNT can be a single-wall carbon nanotube (SWCNT) or a multi-wall carbon nanotube (MWCNT). ${ }^{7}$ It is well known that a SWCNT, depending on its chirality, can be either a metal or a semiconductor. ${ }^{7}$ In-depth investigations have been carried out on various aspects of CNTs. In particular, the field effect characteristic (FEC) of field effect transistors (FETs) made of various kinds of CNTs is an active subject for obvious reason. The FEC of SWCNTs is similar to that of normal metals or semiconductors. However, for MWCNT, ${ }^{8-10}$ things are more complicated.

Measurements on FEC of double-wall carbon nanotubes (DWCNTs), the simplest MWCNTs, have been performed by several groups. ${ }^{11-13}$ Each DWCNT device can experimentally be classified into one of three groups: semiconductor-semiconductor (S-S), metal-metal (M-M) or metal-semiconductor (M-S), and semiconductor-metal (S-M) combinations of two shells of DWCNTs (the first symbol is for the outer shell and the second for the inner one). The S-S and M-M (or M-S) DWCNTs exhibit similar FEC as those of semiconducting and metallic SWCNTs, ${ }^{14}$ respectively. However, the S-M type DWCNTs show FEC with distinct features. (1) In the negative gate voltage region, on-off current ratio can be as large as $10^{1}$ or even $10^{2}$, which is quite different from either those of semiconducting tubes or those of metallic ones. (2) The gate-voltage dependence of source-drain current is no longer exponential in the on-off transition region which is much wider than that in a usual semiconductor device. To explain these features, "proximity effect" was proposed ${ }^{19}$ and qualitatively supported by a twoband tight-binding model on a two-leg ladder. Due to the intershell coupling, the electron wave functions in the inner shell can penetrate into the outer one and, therefore, generate nonzero local density of states (DOS) in the original forbidden gap of the semiconducting shell. Thus, new conducting channels are created and the semiconducting tube becomes weakly conducting. The picture is applicable to all S-M type layer structures. However, the realistic band structures of DWCNTs may affect the proximity effect. It is desirable to calculate the proximity effect with a characteristic band structure of carbon nanotubes (originating from the graphane layers).

Consider a DWCNT FET shown in Fig. 1(a). A sourcedrain voltage $V_{s d}$ is applied between two ends of the outer shell (which is usually called "side contact"), and a back gate voltage $V_{G}$ is applied through the substrate. Thus, electron transport would be mainly through the outer shell when it is metallic because of relatively large intershell tunneling resistance. In our previous two-band ladder tight-binding model,${ }^{19}$ only one band from each shell was considered, and the chiralities and rest bands of a DWCNT were neglected. Thus, those results can only be viewed as a qualitative illustration of proximity effect, instead of a realistic estimate of this effect in DWCNTs. In this Brief Report, we present the detailed calculation of the local DOS on the outer shell of a S-M type of DWCNTs based on a well-known microscopic tight-binding Hamiltonian with its parameters derived from the density-functional theory calculations and/or by fitting them to the experimental measurements. The results provide a direct evidence for metallic proximity effect which can explain well the observed anomalous FEC. ${ }^{13}$

The microscopic tight-binding (TB) Hamiltonian, which includes the coupling of the carbon $\pi$ orbitals within a shell and between two shells, are

$$
H=H_{0}+H_{t},
$$
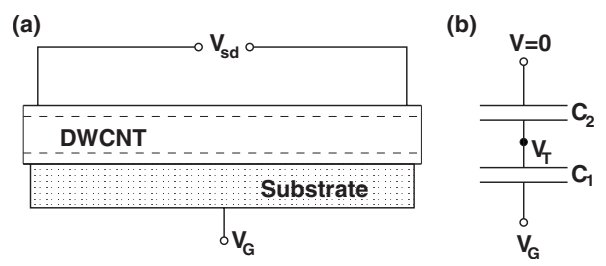

FIG. 1. (a) Schematic illustration of FEC measurement. $V_{s d}$ and $V_{G}$ are source-drain bias and gate voltage, respectively. (b) Simplified sketch of experiment setup. $C_{1}$ is the capacitance between the gate and the DWCNT, and $C_{2}$ is the self-capacitance of the DWCNT. $V_{T}$ is the actual gate potential applied on the tube. 


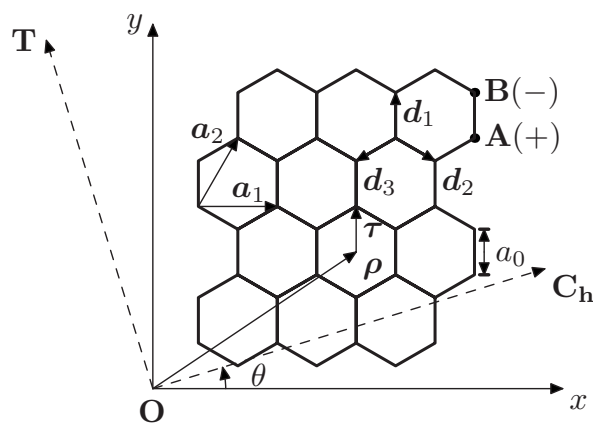

FIG. 2. A piece of graphene lattice. The $x$ and $y$ axes are along the zigzag and armchair directions, while $\mathbf{C}_{\mathbf{h}}$ and $\mathbf{T}$ are along the chiral vector and CNT axis directions, respectively. $\mathbf{a}_{1}$ and $\mathbf{a}_{2}$ are lattice vectors and $\mathbf{d}_{\mathbf{i}}, i=1,2$, and 3 are three nearest-neighbor vectors connecting two sublattices A and B. $\rho$ and $\tau$ are vectors to specify the position of a carbon atom. $\theta$ is the chiral angle and $a_{0}$ is the distance of $\mathrm{C}-\mathrm{C}$ bond.

$$
\begin{gathered}
H_{0}=\sum_{\beta} \sum_{\langle i j\rangle} \gamma_{0} c_{\beta i}^{\dagger} c_{\beta j}, \\
H_{t}=\sum_{i, j} t_{a i, b j} c_{a i}^{\dagger} c_{b j}+\text { H.c.. }
\end{gathered}
$$

$H_{0}$ is a nearest-neighbor (nn) TB Hamiltonian describing uncoupled individual shell. $\beta=a$ and $b$ labels the outer and inner shells, respectively, and $\langle i j\rangle$ denotes nn atoms in each shell. $\gamma_{0} \sim 2.9 \mathrm{eV}$ is the intrashell nn coupling. ${ }^{15}$ $H_{t}$ is the tunneling Hamiltonian between the two shells, the intershell coupling can be assumed to be $t_{a i, b j}$ $=t_{0} \cos \theta_{i j} e^{-\left[d\left(\mathbf{r}_{a i}, \mathbf{r}_{b j}\right)-\Delta\right] / a_{t}}$, where $d\left(\mathbf{r}_{a i}, \mathbf{r}_{b j}\right)$ is the distance between the two atoms on different shells and $\theta_{i j}$ is the angle formed by the two $\pi$ orbitals, and the parameters $t_{0} \sim \gamma_{0} / 3$, $\Delta \sim 0.334 \mathrm{~nm}$, and $a_{t} \sim 0.5 \AA$ are used. By introducing plane-wave basis $c_{\beta j}=\frac{1}{\sqrt{N_{\beta}}} \sum_{\mathbf{k}} e^{i \mathbf{k} \cdot \mathbf{r}_{j}} c_{\beta \eta(j) \mathbf{k}}$, where $\eta= \pm$ labels the two sublattice in graphene and $N_{\beta}$ is the number of graphene unit cells in an individual shell, ${ }^{20,21} H_{0}$ can be rewritten as

$$
H_{0}=\sum_{\beta \eta \mathbf{k}} \gamma_{\mathbf{k}} c_{\beta \eta \mathbf{k}}^{\dagger} c_{\beta-\eta \mathbf{k}},
$$

where $\gamma_{\mathbf{k}}=\gamma_{0} \sum_{j=1}^{3} e^{i \mathbf{k} \cdot \mathbf{d}_{j}}$, and $\mathbf{d}_{j, j=1,2,3}$ are the vectors connecting one carbon atom to the nearest three others (Fig. 2).

The tunneling Hamiltonian under the same Fourier transformation becomes

$$
\begin{aligned}
H_{t}= & \sum_{\mathbf{G}_{a} \mathbf{G}_{b}} \sum_{\mathbf{k}_{a} \mathbf{k}_{b}} \sum_{\eta_{a} \eta_{b}} e^{i\left[\mathbf{G}_{a} \cdot\left(\rho_{a}+\eta_{a} \tau_{a}\right)-\mathbf{G}_{b} \cdot\left(\rho_{b}+\eta_{b} \tau_{b}\right)\right]} \\
& \times t_{\mathbf{k}_{a}+\mathbf{G}_{a}, \mathbf{k}_{b}+\mathbf{G}_{b}} c_{\eta_{a} \mathbf{k}_{a}}^{\dagger} c_{\eta_{b} \mathbf{k}_{b}}+\text { H.c. }
\end{aligned}
$$

where $\mathbf{G}$ is the reciprocal lattice of graphene, $\rho_{\beta}+\eta_{\beta} \tau_{\beta}$ specifies the location of a carbon atom in graphene, and

$$
t_{\mathbf{k}_{a}, \mathbf{k}_{b}}=\frac{1}{A_{c e l l}^{2} \sqrt{N_{a} N_{b}}} \int d \mathbf{r}_{a} d \mathbf{r}_{b} e^{i\left(\mathbf{k}_{b} \cdot \mathbf{r}_{b}-\mathbf{k}_{a} \cdot \mathbf{r}_{a}\right)} t_{a i, b j} .
$$

These are the theoretical preparations for our numerical calculation.

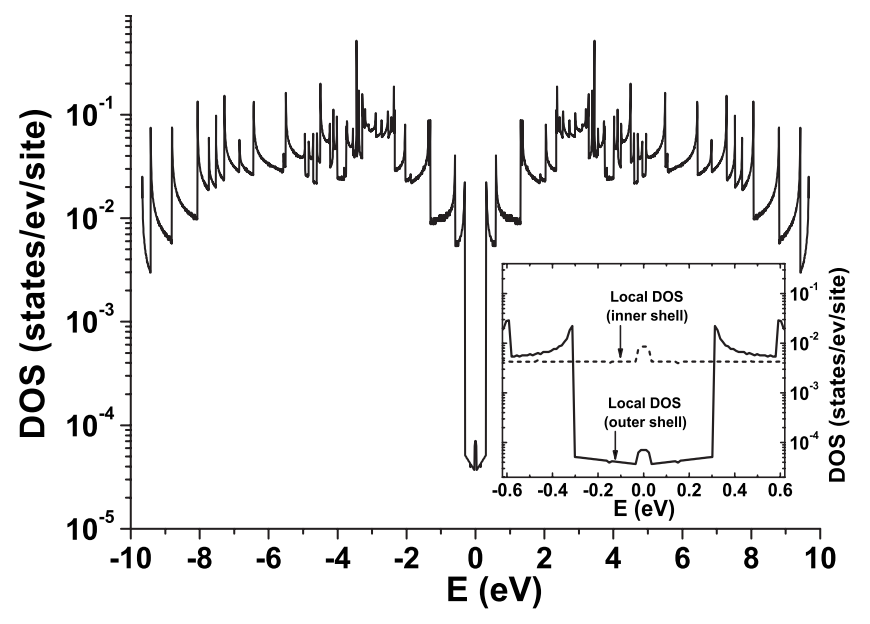

FIG. 3. The local DOS on the outer shell of $(9,0) @(17,0)$ DWCNT at $V_{G}=0$. A nonzero DOS appears at the original forbidden gap of the $(17,0)$ shell due to proximity effect. Inset: Small peaks in local DOS of both $(17,0)$ and $(9,0)$ shells are observed around $E=0$, which may be due to the nonperiodicity of the intershell coupling.

In real experiments, the chiralities of the two shells of a DWCNT have numerous possible combinations. It is impossible to perform calculations for all possible chirality combinations. For simplicity, we use CNTs with chiral indices $(n, 0)$ to mimic the two shells of the S-M type DWCNT. This is a specific choice of a DWCNT, but the physics discussed here does not depend on the specificity of the system. The intershell distance of DWCNT is $\Delta \sim 0.334 \mathrm{~nm},{ }^{7}$ and the indices should satisfy $n_{\text {out }}-n_{\text {in }} \simeq 8$. Considering the dimension of the real DWCNTs used in experiments, we choose $(9,0) @(17,0)$ DWCNT in our calculation $[(9,0)$ are the indices of the inner shell and $(17,0)$ are for the outer one]. $1 \times 10^{4}$ sample points in the first Brillouin zone are used to ensure adequate accuracy.

Proximity effect predicts a nonzero local DOS on the outer shell of a S-M type DWCNT as long as the intershell coupling exists and regandless of whether with or without a gate voltage. The local DOS on the outer shell in the absence of a gate voltage is plotted in Fig. 3. Each main spike corresponds to an edge of a subband of the original $(17,0)$ semiconducting SWCNT, while the secondary spikes come from the penetration of the DOS of the inner $(9,0)$ shell. A nonzero DOS appears in the original gap region with an order of $10^{-5}-10^{-4}$ states $/ \mathrm{eV}$ per site. It is this finite local DOS which results in the anomalous FECs observed in S-M type DWCNTs as explained in Ref. 19.

In addition, small bumps appear around $E=0$ with a width of about $0.06 \mathrm{eV}$ for the local DOS of both inner and outer shells, as shown in the inset of Fig. 3. As the intershell coupling increases, the width and height of these peaks increase. This feature does not exist for SWCNTs. It is known ${ }^{22}$ that both randomness and quasiperiodic coupling can dramatically alter the spectrum of a low dimensional system. In the one-dimensional case with random coupling, $E=0$ is a 


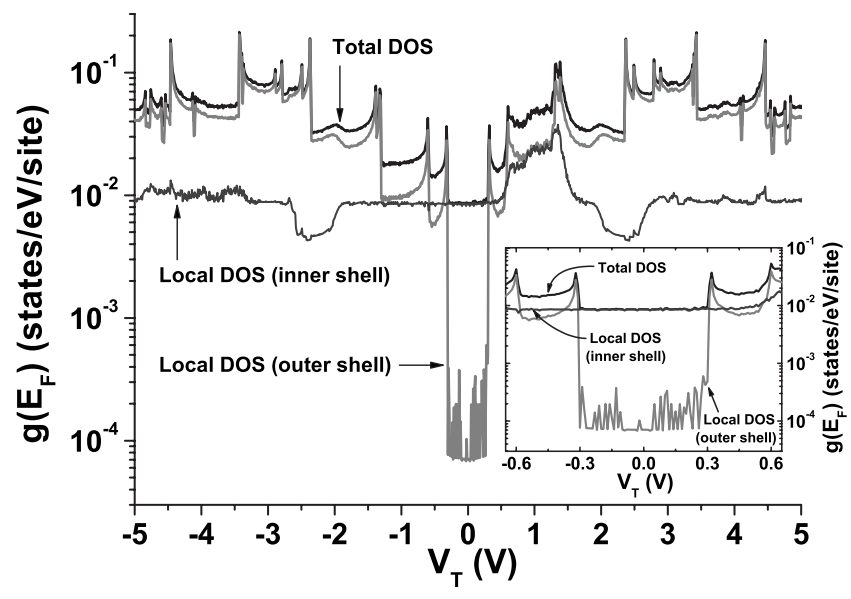

FIG. 4. Local DOS on the outer shell of $(9,0) @(17,0)$ DWCNT at the Fermi level of the electrodes as a function of gate voltage. The inset is the enlarged part at low gate voltage.

special point. ${ }^{22}$ The appearance of states of $E=0$ in the quasirandom intershell coupling is due to the bipartite lattice of the Dirac-electron system of graphene near the Fermi surface. It is known that such a system has supersymmetry that can have $E=0$ edge states or disordered states in the presence of an edge and/or disorders, a subject of recent attention in graphene physics. ${ }^{23,24}$ Since these states are highly localized, they should not participate in the electron transport. These bumps are the consequences of the combined effects of the nonperiodicity of the intershell coupling and the Diracelectron nature of graphane. However, further theoretical and experimental verifications are needed. Low-temperature scanning tunneling microscopy may be a useful probe to test the result.

To understand the observed anomalous ${ }^{11-13}$ FEC in DWCNT FETs, one needs to see how the strength of the proximity effect varies under a gate voltage. Thus, we shall calculate the gate-voltage dependence of local DOS on the semiconductor shell, especially the DOS around the Fermi level. As sketched in Fig. 1, since the voltage is applied via the back gate (substrate), the actual voltage on the DWCNT is only a portion of the total applied voltage. ${ }^{25}$ The percentage $\beta$ is related to the mutual capacitance $C_{1}$ (between the substrate and the DWCNT) and the self-capacitance of DWCNT $C_{2}$ as $\beta=C_{1} /\left(C_{1}+C_{2}\right)$. Thus, the on-site energies of the DWCNT vary with the gate voltage $V_{G}$ by an amount of $V_{T}=\beta V_{G}$. Due to the Fermi level pinning, the gate voltage falls mainly on the outer shell, and the gate voltage will shift the original energy spectrum of the outer shell as explained in Ref. 19. For the case of small source-drain voltage, the electrochemical potential gradient inside the DWCNT can be neglected, and the Fermi levels $\left(E_{F}\right)$ of both electrodes can be assumed to be the same $\left(E_{F}=0\right.$, without losing generality). With all the above assumptions, the term $\Sigma_{i} V_{T} c_{a i}^{\dagger} c_{a i}$ due to the gate voltage is added to Eq. (1). The $V_{T}$ dependence of the total DOS and the local DOS on both the outer and inner shells at the Fermi levels $g\left(E_{F}\right)$ are plotted in Fig. 4. In our numerical simulation, we shift the original energy spectrum of the outer shell with the value $-e \beta V_{G}$ to take into account the effect of the gate voltage.

In real experiments, parameter $\beta$ is about 0.1 or smaller, ${ }^{13}$ and the gate voltage is usually tens of $\mathrm{eV}$. Thus, $V_{T}$ is chosen in the range of $-5 \mathrm{~V} \leqslant V_{T} \leqslant 5 \mathrm{~V}$ in our calculation. The following features can be seen from Fig. 4. (1) Both the local DOS on the inner and outer shells are roughly symmetric for positive and negative gate voltages, except for some secondary structures, especially those within $\left|V_{T}\right| \leqslant 1.8 \mathrm{~V}$. The rough overall symmetry comes from the symmetric energy band structures of individual SWCNTs about zero energy, while the small asymmetry is due to the quasirandom intershell coupling. Since this coupling is generally much smaller than the bandwidth of graphene, their effects are secondary and affect original gap states more than others. (2) When $V_{G}$ varies, the local DOS on the inner shell is roughly fixed with a value of around $0.008-0.01 \mathrm{states} / \mathrm{eV}$ per site. (3) The curves in Figs. 3 and 4 are quite similar. This is because the local DOS on the outer shell at the Fermi level traces the band structure of the outer $(17,0)$ shell when gate voltage $V_{G}$ is applied (either negative or positive) since the bands of the outer shell move upward (downward) with a negative (positive) gate voltage. (4) The secondary features around $-0.3 \mathrm{~V}<V_{T}<0.3 \mathrm{~V}$ (the inset of Fig. 4) are not numerical errors. Their origins may be due to the nonperiodicity of the intershell coupling. (5) For large positive gate voltage, numerical result shows an enhancement in local DOS on the outer shell, but this may be hard to verify in a real experiment with $p$-type CNTs. (6) $g\left(E_{F}\right)$ in the absence of the gate voltage $\left(V_{G}=0\right)$ is of the order of $10^{-4}-10^{-5}$ states/eV per site. However, it increases to the order of $10^{-2}$ states/eV per site with a large negative $V_{G}$. Under a small source-drain bias and at a low temperature, the source-drain current $I_{s d}$ should be proportional to $g\left(E_{F}\right)$. Thus, the gate-voltage dependence of $g\left(E_{F}\right)$ explains well the observed on-off current ratio of $10^{2}-10^{3}$ in experiments.

The above DOS calculations are based on the microscopic tight-binding Hamiltonian (1). This Hamiltonian catches the main physics of electron transport in DWCNTs, but it has also some limitations. First, temperature effect has not been considered in this model. Thus, we can only compare our numerical calculations with the experimental results at extremely low temperature. Second, the curvatures of CNTs are not considered in our calculations. Large curvature of small SWCNTs leads to a hybridization of $\sigma^{*}$ and $\pi^{*}$ orbitals that results in some modifications of the original band structures of graphene. Consequently, tube curvature may affect the strength of the proximity effect. Third, electron-electron (e-e) interactions are neglected in this model. e-e interaction, which can also modify the band structures of CNTs, should be large for small CNTs. All the above neglected factors may affect the actual strength of the proximity effect, but they shall not change the physics revealed here. Last, but not least, we would like to emphasize that in this work we attribute the observed anomalous FEC in DWCNTs to the proximity effect, though the proximity effect itself is a wellknown phenomenon in heterostructures.

In conclusion, detailed local DOS of the S-M type DWCNT is calculated by using a well-known microscopic 
tight-binding Hamiltonian. The results show direct evidence of the existence of metallic proximity effect. The gatevoltage dependence of the local DOS can explain some of the anomalous FECs of S-M type DWCNTs observed in experiments.
We would like to thank X. R. Wang for his substantial contributions to this work and for his generosity of not being a coauthor. This work is supported by UGC, Hong Kong, through RGC CERG Grants No. HKUST6067/02P and No. 603904 .
${ }^{1}$ Supriyo Datta, Electronic Transport in Mesoscopic Systems (Cambridge University Press, Cambridge, England, 1997).

${ }^{2}$ B. J. van Wees, H. van Houten, C. W. J. Beenakker, J. G. Williamson, L. P. Kouwenhoven, D. van der Marel, and C. T. Foxon, Phys. Rev. Lett. 60, 848 (1988); P. Poncharal, Z. L. Wang, D. Ugarte, and W. A. deHeer, Science 283, 1513 (1999); C. W. J. Beenakker and H. van Houten, in Solid State Physics, edited by H. Ehrenreich and D. Turnbull (Academic, New York, 1991).

${ }^{3}$ S. Iijima and T. Ichihashi, Nature (London) 363, 603 (1993).

${ }^{4}$ R. Saito, G. Dresselhaus, and M. S. Dresselhaus, Physical Properties of Carbon Nanotubes (Imperial College, London, 1998).

${ }^{5}$ Z. Yao, H. W. Ch. Postma, L. Balents, and C. Dekker, Nature (London) 402, 273 (1999).

${ }^{6}$ X. R. Wang, Yupeng Wang, and Z. Z. Sun, Phys. Rev. B 65, 193402 (2002); S. Yin, Z. Z. Sun, J. Lu, and X. R. Wang, Appl. Phys. Lett. 88, 233110 (2006); Z. K. Tang and X. R. Wang, ibid. 68, 3449 (1996).

${ }^{7}$ M. S. Dresselhaus, G. Dresselhaus, and P. C. Eklund, Science of Fullerenes and Carbon Nanotubes (Academic, London, 1995); R. Saito, G. Dresselhaus, and M. S. Dresselhaus, Physical Properties of Carbon Nanotubes (Imperial College Press, London, 1998).

${ }^{8}$ S. Frank, P. Poncharal, Z. L. Wang, and W. A. de Heer, Science 280, 1744 (1998).

${ }^{9}$ A. Urbina, I. Echeverría, A. Pérez-Garrido, A. Díaz-Sánchez, and J. Abellán, Phys. Rev. Lett. 90, 106603 (2003).

${ }^{10}$ S. Sanvito, Y.-K. Kwon, D. Tománek, and C. J. Lambert, Phys. Rev. Lett. 84, 1974 (2000).

${ }^{11}$ R. Martel, T. Schimidt, H. R. Shea, T. Hertel, and Ph. Avouris, Appl. Phys. Lett. 73, 2447 (1998).
${ }^{12}$ T. Shimada, T. Sugai, Y. Ohno, S. Kishimoto, T. Mizutani, H. Yoshida, T. Okazaki, and H. Shinohara, Appl. Phys. Lett. 84, 2412 (2004).

${ }^{13}$ S. Wang, X. L. Liang, Q. Chen, Z. Y. Zhang, and L.-M. Peng, J. Phys. Chem. B 109, 17361 (2005).

${ }^{14}$ S. J. Tans, A. R. M. Verschueren, and C. Dekker, Nature (London) 393, 49 (1998).

${ }^{15}$ S. Roche, F. Triozon, A. Rubio, and D. Mayou, Phys. Rev. B 64, 121401(R) (2001); Y. Miyamoto, S. Saito, and D. Tománek, ibid. 65, 041402(R) (2001); S. Uryu, ibid. 69, 075402 (2004).

${ }^{16}$ A. Javey, J. Guo, Q. Wang, M. Lundstrom, and H. J. Dai, Nature (London) 424, 654 (2003).

${ }^{17}$ L. Chico, V. H. Crespi, L. X. Benedict, S. G. Louie, and M. L. Cohen, Phys. Rev. Lett. 76, 971 (1996); C. T. White and T. N. Todorov, Nature (London) 393, 240 (1998).

${ }^{18}$ S. J. Tans, M. H. Devoret, H. J. Dai, A. Thess, R. E. Smalley, L. J. Geerligs, and C. Dekker, Nature (London) 386, 474 (1997).

${ }^{19}$ J. Lu, S. Yin, L. M. Peng, Z. Z. Sun, and X. R. Wang, Appl. Phys. Lett. 90, 052109 (2007).

${ }^{20}$ A. A. Maarouf, C. L. Kane, and E. J. Mele, Phys. Rev. B 61, 11156 (2000).

${ }^{21}$ S. Wang and M. Grifoni, Phys. Rev. Lett. 95, 266802 (2005).

${ }^{22}$ W. L. Chan, X. R. Wang, and X. C. Xie, Phys. Rev. B 54, 11213 (1996); X. R. Wang, ibid. 51, 9310 (1995); 53, 12035 (1996).

${ }^{23}$ M. Ezawa, Phys. Rev. A (to be published); Y.-W. Son, M. L. Cohen, and S. G. Louie, Nature (London) 444, 347 (2006).

${ }^{24}$ W. Yao, D. Xiao, and Q. Niu, arXiv:0705.4683v2 (unpublished).

${ }^{25}$ S. D. Wang, Z. Z. Sun, N. Cue, H. Q. Xu, and X. R. Wang, Phys. Rev. B 65, 125307 (2002). 